\title{
Exact relativistic $\beta$ decay endpoint spectrum
}

\author{
S. S. Masood, ${ }^{1, *}$ S. Nasri ${ }^{2, \dagger}$ J. Schechter, ${ }^{3, \ddagger}$ M. A. Tórtola, ${ }^{4, \S}$ J. W. F. Valle, ${ }^{5, \|}$ and C. Weinheimer ${ }^{6, \uparrow}$ \\ ${ }^{1}$ Department of Physics, 2700 Bay Area Boulevard, University of Houston Clear Lake, Houston, Texas 77058, USA \\ ${ }^{2}$ Institute for Fundamental Theory, University of Florida, Gainesville, Florida, USA \\ ${ }^{3}$ Department of Physics, Syracuse University, Syracuse, New York 13244-1130, USA \\ ${ }^{4}$ Departamento de Física and CFTP, Instituto Superior Técnico, Avenida Rovisco Pais 1, P-1049-001 Lisboa, Portugal \\ ${ }^{5}$ AHEP Group, Institut de Física Corpuscular-C.S.I.C./Universitat de València, Edificio Institutos de Paterna, Apartado 22085, \\ E-46071 Valencia, Spain \\ ${ }^{6}$ Institut für Kernphysik, Westfälische Wilhelms-Universität Münster, Wilhelm-Klemm-Strasse 9, D-48149 Münster, Germany
}

(Received 13 June 2007; published 5 October 2007)

\begin{abstract}
The exact relativistic form for the $\beta$ decay endpoint spectrum is derived and presented in a simple factorized form. We show that our exact formula can be well approximated to yield the endpoint form used in the fit method of the KATRIN Collaboration. We also discuss the three-neutrino case and how information from neutrino oscillation experiments may be useful in analyzing future $\beta$ decay endpoint experiments.
\end{abstract}

DOI: 10.1103/PhysRevC.76.045501

PACS number(s): 14.60.Pq, 13.30.-a, 23.40.-s, 23.40.Bw

\section{INTRODUCTION}

The discovery of neutrino oscillations [1-5] probes the neutrino squared mass differences and mixing angles [6], but it leaves open the issue of the absolute scale of neutrino mass. The latter has important cosmological implications in the cosmic microwave background and large-scale structure in the Universe, as already indicated by the sensitivities reached, for example, by the recent WMAP-3 [7], the 2dF Galaxy Redshift Survey [8], and Sloan Digital Sky Survey results [9]. One expects even better sensitivities in the next generation of cosmological observations [10,11]. Interesting as these may be, there are essentially only two ways to get insight into the absolute scale of neutrino mass in the laboratory: searches for neutrinoless double $\beta$ decay [12] and investigations of the $\beta$ spectra near their endpoints [13-18]. For the latter direct search for the neutrino mass a very low $\beta$ endpoint is crucial: Tritium was used in the most sensitive spectrometer experiments $[14,15]$ and rhenium in the upcoming cryobolometer experiments [19].

Currently, a next-generation tritium $\beta$ decay experiment is being prepared, scaling up the size and precision of previous experiments by an order of magnitude, and increasing the intensity of the tritium $\beta$ source: the KArlsruhe TRItium Neutrino experiment KATRIN [13,16-18]. Such an improvedsensitivity experiment will probe neutrino masses an order of magnitude smaller than the current limits and therefore play a crucial role in probing for direct effects of neutrino masses.

Prompted by the prospects that high sensitivities can be achieved in the next generation of high-precision neutrino mass searches from tritium $\beta$ decay experiments $[16,17]$ we

\footnotetext{
*masood@uhcl.edu

†snasri@ufl.edu

†schechte@phy.syr.edu

§mariam@cftp.ist.utl.pt

"valle@ific.uv.es

ๆweinheimer@uni-muenster.de
}

reexamine the accuracy of the kinematical formulas used in the determination of neutrino masses from the shape of the endpoint spectrum. We also discuss the interplay of neutrino oscillation data and the expectations for the $\beta$ decay endpoint counting rates for the different types of neutrino mass spectra.

\section{RELATIVISTIC $\beta$ DECAY KINEMATICS}

In what follows we label the relativistic momenta and energies involved in tritium $\beta$ decay according to

$$
{ }^{3} \mathrm{H}(\mathbf{0}, M) \rightarrow{ }^{3} \mathrm{He}^{+}\left(\mathbf{p}^{\prime}, E^{\prime}\right)+e^{-}\left(\mathbf{p}_{\mathbf{e}}, E_{e}\right)+\overline{v_{e}}\left(\mathbf{p}_{v}, E_{v}\right) .
$$

The masses of ${ }^{3} \mathrm{He}^{+}, e^{-}$, and $\bar{v}_{e}$ are denoted by $M^{\prime}, m_{e}$, and $m_{v}$, respectively. To see the convenience of an exact relativistic description we mention, as recently noted in Ref. [20], that the well-known relativistic formula for the maximum electron energy

$$
E_{e}^{\max }=\frac{1}{2 M}\left[M^{2}+m_{e}^{2}-\left(m_{v}+M^{\prime}\right)^{2}\right],
$$

gives a value about $3.4 \mathrm{eV}$ lower than the approximation $M-M^{\prime}-m_{v}$ often used. This suggests the desirability of carrying out the full phase-space integration using relativistic kinematics.

Start from the standard formula for the decay width at rest,

$$
\begin{aligned}
\Gamma= & \frac{1}{2^{9} \pi^{5} M} \int \frac{d^{3} p_{e} d^{3} p_{\nu} d^{3} p^{\prime}}{E_{e} E_{v} E^{\prime}} \\
& \times|\mathcal{M}|^{2} \delta^{4}\left(p_{\text {initial }}-p^{\prime}-p_{e}-p_{v}\right),
\end{aligned}
$$

where $|\mathcal{M}|^{2}$ denotes the spin-summed, Lorentz-invariant "squared" amplitude. To explore the constraint of Lorentz invariance one might a priori consider expanding $|\mathcal{M}|^{2}$ in terms of invariants constructed from the four-momenta. For example, up to two powers of momenta, the most general form is

$$
|\mathcal{M}|^{2}=A-B p_{e} \cdot p_{v}-C p^{\prime} \cdot p_{\text {initial }}+\cdots,
$$


where $A, B$, and $C$ are constants. Now it is easy to perform some initial integrations. As usual $\int d^{3} p^{\prime}$ is first done with the momentum delta function. Then the angle between $\mathbf{p}_{e}$ and $\mathbf{p}_{v}$ is eliminated by using the energy delta function. Three more angular integrals are trivial. As a result one may replace in the ${ }^{3} \mathrm{H}$ rest frame

$|\mathcal{M}|^{2} \rightarrow A+B\left(E_{e} E_{v}-\mathbf{p}_{\mathbf{e}} \cdot \mathbf{p}_{v}\right)+C M\left(M-E_{e}-E_{v}\right)$,

where

$\mathbf{p}_{\mathbf{e}} \cdot \mathbf{p}_{v} \equiv \frac{1}{2}\left[M^{2}-M^{\prime 2}+m_{e}^{2}+m_{v}^{2}-2 M E_{e}+2 E_{v}\left(E_{e}-M\right)\right]$.

Equation (5) can now be inserted in the resulting usual formula [21]

$$
\Gamma=\frac{1}{2^{6} \pi^{3} M} \int d E_{\nu} d E_{e}|\mathcal{M}|^{2} .
$$

Next we find $d \Gamma / d E_{e}$ by integrating over $d E_{v}$ for each $E_{e}$. The limits of integration $E_{v}^{\min }\left(E_{e}\right)$ and $E_{v}^{\max }\left(E_{e}\right)$ can be read from Ref. [21]. The most tedious part of the present calculation is finding the factorizations:

$$
\begin{aligned}
E_{v}^{\max }-E_{v}^{\min }= & \frac{2 M p_{e}}{\left(m_{12}\right)^{2}}\left(E_{e}^{\max }-E_{e}\right)^{1 / 2} \\
& \times\left[E_{e}^{\max }-E_{e}+\frac{2 m_{v} M^{\prime}}{M}\right]^{1 / 2}, \\
E_{v}^{\max }+E_{v}^{\min }= & \frac{2 M}{\left(m_{12}\right)^{2}}\left(M-E_{e}\right) \\
& \times\left[E_{e}^{\max }-E_{e}+\frac{m_{v}}{M}\left(M^{\prime}+m_{v}\right)\right],
\end{aligned}
$$

wherein

$$
\left(m_{12}\right)^{2}=M^{2}-2 M E_{e}+m_{e}^{2} .
$$

The importance of the factorization is that it makes the behavior at the endpoint $E_{e}=E_{e}^{\max }$ transparent. Then we have the exact relativistic result,

$$
\begin{aligned}
\frac{d \Gamma}{d E_{e}}= & \frac{1}{(2 \pi)^{3}} \frac{p_{e}}{4\left(m_{12}\right)^{2}} \sqrt{y\left(y+\frac{2 m_{v} M^{\prime}}{M}\right)}\left\{A+C M\left(M-E_{e}\right)\right. \\
& +B M \frac{M E_{e}-m_{e}^{2}}{\left(m_{12}\right)^{2}}\left[y+\frac{m_{v}}{M}\left(M^{\prime}+m_{v}\right)\right] \\
& \left.-C \frac{M^{2}}{\left(m_{12}\right)^{2}}\left(M-E_{e}\right)\left[y+\frac{m_{v}}{M}\left(M^{\prime}+m_{v}\right)\right]\right\},
\end{aligned}
$$

where $y=E_{e}^{\max }-E_{e}$.

As it stands, this formula is based only on the kinematical assumption in Eq. (4). It obviously vanishes at the endpoint $y=0$ as $\sqrt{y}$. Note that all other terms are finite at $y=0$. The overall factor $\sqrt{y\left(y+2 m_{v} M^{\prime} / M\right)}$ gives the behavior of $\frac{d \Gamma}{d E_{e}}$ extremely close to $y=0$ for any choice of $A, B$, and $C$ but departs from $\frac{d \Gamma}{d E_{e}}$ away from the endpoint.

Dynamics is traditionally put into the picture [22] by examining the spin sum for a four-fermion interaction wherein the nuclear matrix element is assumed constant. This is presented as a non-Lorentz-invariant term,

$$
|\mathcal{M}|^{2}=B E_{e} E_{v} .
$$

We will see that this is excellently approximated in our fully relativistic model by

$$
A=C=0, \quad B \neq 0 .
$$

A more accurate treatment of the underlying interaction might give rise to small admixtures of nonzero $A$ and $C$ as well as other unwritten coefficients in Eq. (4).

The form for the spectrum shape near the endpoint that results from putting $A=C=0$ in Eq. (11) is

$$
\begin{aligned}
\frac{d \Gamma}{d E_{e}}= & \frac{p_{e} M B}{(2 \pi)^{3} 4\left(m_{12}\right)^{4}}\left(M E_{e}-m_{e}^{2}\right) \sqrt{y\left(y+\frac{2 m_{v} M^{\prime}}{M}\right)} \\
& \times\left[y+\frac{m_{v}}{M}\left(M^{\prime}+m_{v}\right)\right] .
\end{aligned}
$$

Note that if we had employed the nonrelativistic form given in Eq. (12) the net result would be a replacement of an overall factor in Eq. (14) according to

$$
\left(M E_{e}-m_{e}^{2}\right) \rightarrow\left(M E_{e}-E_{e}^{2}\right) .
$$

The difference of these two factors yields the contribution of the $\mathbf{p}_{\mathbf{e}} \cdot \mathbf{p}_{v}$ term. It is really negligible near the endpoint region because it is proportional to $p_{e}^{2}$ and is suppressed like $p_{e}^{2} /\left(M E_{e}\right)$ compared to unity. We have checked that the result of our calculation with just the $E_{e} E_{v}$ term agrees with the calculation of Ref. [23], though their result looks much more complicated, as they did not present it in the simpler factorized form given here.

Note that only the two rightmost factors vary appreciably near the endpoint of Eq. (14). If we further approximate $M^{\prime} / M \rightarrow 1$ and $\frac{M^{\prime}+m_{v}}{M} \rightarrow 1$ the endpoint shape is well described by

$$
\frac{d \Gamma}{d E_{e}} \propto\left(y+m_{\nu}\right) \sqrt{y\left(y+2 m_{\nu}\right)} .
$$

Now we compare with the formula used in the experimental analysis [15]

$$
\frac{d \Gamma}{d E_{e}} \propto\left(E_{0}-V_{i}-E\right) \sqrt{\left(E_{0}-V_{i}-E\right)^{2}-m_{v}^{2}} .
$$

This agrees with the approximation in Eq. (16) if one identifies

$$
\left(E_{0}-V_{i}-E\right)=y+m_{\nu} .
$$

Note that $E$ is the nonrelativistic energy given by $E=E_{e}-$ $m_{e}$. Furthermore, $E_{0}-V_{i}$ is identified with our $\left(M-M^{\prime}-\right.$ $\left.m_{e}-\delta E_{e}^{\max }\right)$, with $\delta E_{e}^{\max }$ defined by

$$
E_{e}^{\max }=M-M^{\prime}-m_{v}-\delta E_{e}^{\max },
$$

which was shown in Ref. [20] to be independent of $m_{v}$ to a good approximation. Thus we see that the exact relativistic endpoint structure obtained here may be well approximated by the form used in the experimental analysis.

Often, authors express results in terms of a variable $x$, which from our discussion may be seen to be the same as

$$
x=-y-m_{\nu}=E_{e}-E_{e}^{\max }-m_{\nu} .
$$

In Fig. $1, d \Gamma / d E_{e}$ as computed from the exact formula, Eq. (14), is compared with its approximate analog as a function 


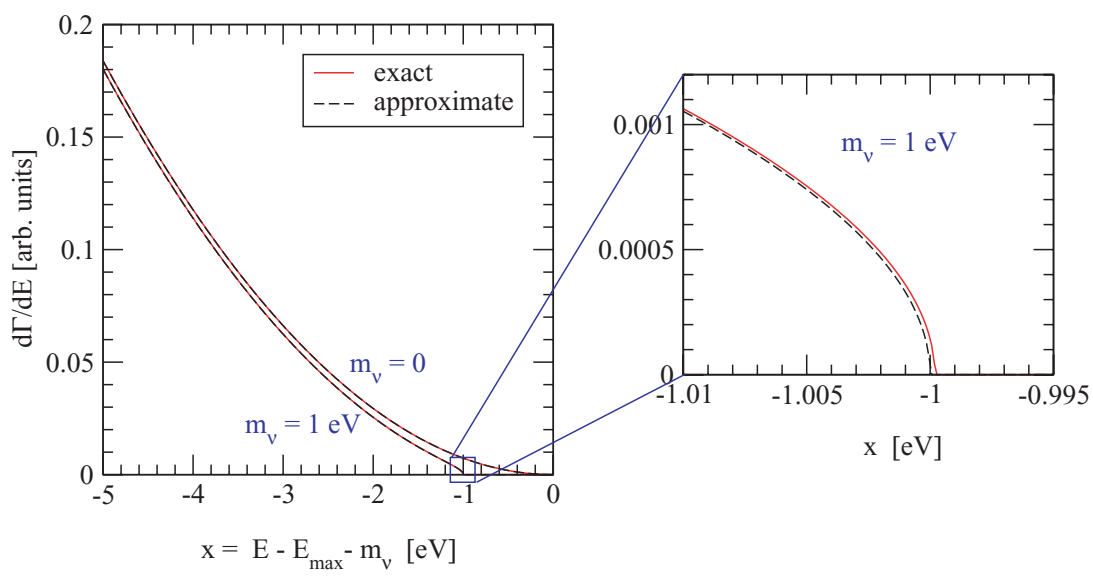

FIG. 1. (Color online) Comparison of approximate and exact formula.

of $x$. As can be seen, the differences between the approximate and exact formulas are tiny.

It may be worthwhile to remark that the exact relativistic kinematical expression in Eq. (14) is no more complicated than the approximation one ordinarily uses.

\section{NUMERICAL SIMULATIONS}

We have done Monte Carlo simulations by creating random data sets following the exact relativistic kinematical expression in Eq. (14) for neutrino masses of $m_{v}=0 \mathrm{eV}$ and $m_{v}=$ $1 \mathrm{eV}$ and fitting them with the standard formula, Eq. (17). The simulations were performed for a KATRIN-like experiment [18] by considering

(i) the rovibrational states of the $T_{2} \rightarrow\left(T^{3} \mathrm{He}\right)^{+}$decay [24];

(ii) a signal rate from a KATRIN-like molecular gaseous windowless tritium source with a column density of $5 \times$ $10^{17}$ molecules $/ \mathrm{cm}^{2}$ over an active area of $53 \mathrm{~cm}^{2}$ and an accepted solid angle of $\Delta \Omega / 4 \pi=0.18$;

(iii) an expected background rate of $0.01 \mathrm{~s}^{-1}$;

(iv) a response function of a KATRIN-like experiment considering the energy losses within the tritium source and the main spectrometer transmission function with a total width of $0.93 \mathrm{eV}$; and

(v) three years of total data, covering an energy range of the $25 \mathrm{eV}$ below and $5 \mathrm{eV}$ above the tritium endpoint following an optimized measurement point distribution [18].

Figure 2 shows the results for the observable $m_{v}^{2}$ obtained from the fitting of 10,000 sets of Monte Carlo data randomized according to the exact relativistic formula [Eq. (14)] and to a fitting routine using the standard formula [Eq. (17)], with neutrino masses assumed to be $0 \mathrm{eV}$ (left) and $1 \mathrm{eV}$ (right). The rms values of the Gaussian-like distributions correspond to the expected statistical uncertainty $\Delta m_{v \text {,stat }}^{2}$ for a KATRIN-like experiment. Clearly the mean value of the fit results for the neutrino mass squared, $m_{v}^{2}$, does not show any significant deviation from the starting assumption of $m_{v}=$ $0 \mathrm{eV}$ or $m_{v}=1 \mathrm{eV}$, respectively. This establishes that the exact relativistic formula, Eq. (14), can be well approximated by the standard equation (17) for the precision needed for the nextgeneration tritium experiment KATRIN. The validity of this approximation is probably because KATRIN is investigating the last $25 \mathrm{eV}$ of the $\beta$ spectrum below its endpoint only, where the recoil corrections are nearly independent of the electron energy.

\section{THREE-NEUTRINO CASE}

Of course, the most interesting application is to the case of three neutrinos with different masses, $m_{1}, m_{2}$, and $m_{3}$. Then there will be different endpoint energies $E_{i}^{\max }$ corresponding
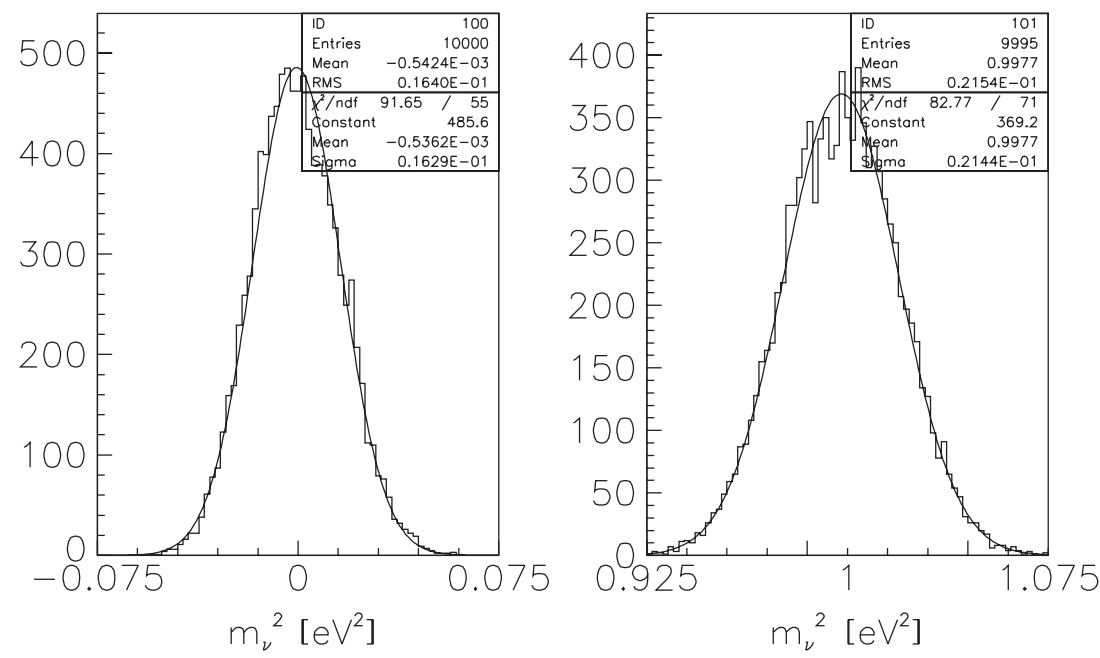

$045501-3$
FIG. 2. Results on the observable $m_{v}^{2}$ from the fitting of 10,000 sets of Monte Carlo data randomized according to the exact relativistic formula [Eq. (14)] and to a fitting routine based on the standard formula [Eq. (17)] for neutrino masses of $0 \mathrm{eV}$ (left) and $1 \mathrm{eV}$ (right). The rms values of the Gaussian-like distributions correspond to the expected statistical uncertainty $\Delta m_{v, \text { stat }}^{2}$ for a KATRIN-like experiment. 
to each one. The effective endpoint factor in the good approximation of Eq. (16) is the weighted sum,

$$
F_{\mathrm{eff}}\left(E_{e}\right)=\sum_{i=1}^{3}\left|K_{1 i}\right|^{2}\left(y_{i}+m_{i}\right)\left[y_{i}\left(y_{i}+2 m_{i}\right)\right]^{1 / 2} \theta\left(y_{i}\right),
$$

where $y_{i}\left(E_{e}\right)=E_{i}^{\max }-E_{e}$ and the $K_{1 i}$ are the elements of the $3 \times 3$ lepton mixing matrix $[25,26]$. We note that the further good approximation that the quantity $\delta E_{e}^{\max }$ is independent of the neutrino mass gives the useful relation

$$
y_{i}-y_{j}=m_{j}-m_{i}
$$

Now let the unindexed quantity $y$ stand for the $y_{i}$ with the smallest of the neutrino masses. Using Eq. (22) allows us to write the explicit formula for the case (denoted "normal hierarchy," or NH) where $m_{1}$ is the lightest of the three neutrino masses as

$$
\begin{aligned}
F_{\mathrm{NH}}(y)= & \left|K_{11}\right|^{2}\left(y+m_{1}\right)\left[y\left(y+2 m_{1}\right)\right]^{1 / 2} \\
& +\left|K_{12}\right|^{2}\left(y+m_{1}\right)\left[\left(y+m_{1}-m_{2}\right)\right. \\
& \left.\times\left(y+m_{1}+m_{2}\right)\right]^{1 / 2} \theta\left(y+m_{1}-m_{2}\right) \\
& +\left|K_{13}\right|^{2}\left(y+m_{1}\right)\left[\left(y+m_{1}-m_{3}\right)\right. \\
& \left.\times\left(y+m_{1}+m_{3}\right)\right]^{1 / 2} \theta\left(y+m_{1}-m_{3}\right) .
\end{aligned}
$$

In the other case of interest (denoted "inverse hierarchy," or IH) we have

$$
\begin{aligned}
F_{\mathrm{IH}}(y)= & \left|K_{13}\right|^{2}\left(y+m_{3}\right)\left[y\left(y+2 m_{3}\right)\right]^{1 / 2} \\
& +\left|K_{11}\right|^{2}\left(y+m_{3}\right)\left[\left(y+m_{3}-m_{1}\right)\right. \\
& \left.\times\left(y+m_{3}+m_{1}\right)\right]^{1 / 2} \theta\left(y+m_{3}-m_{1}\right) \\
& +\left|K_{12}\right|^{2}\left(y+m_{3}\right)\left[\left(y+m_{3}-m_{2}\right)\right. \\
& \left.\times\left(y+m_{3}+m_{2}\right)\right]^{1 / 2} \theta\left(y+m_{3}-m_{2}\right),
\end{aligned}
$$

where $m_{3}$ is the lightest of the three neutrino masses. From these equations we may easily find the counting rate in the energy range from the appropriate endpoint up to $y_{\max }$ as proportional to the integral

$$
n_{\mathrm{NH}}\left(y_{\max }\right)=\int_{0}^{y_{\max }} d y F_{\mathrm{NH}}(y)
$$

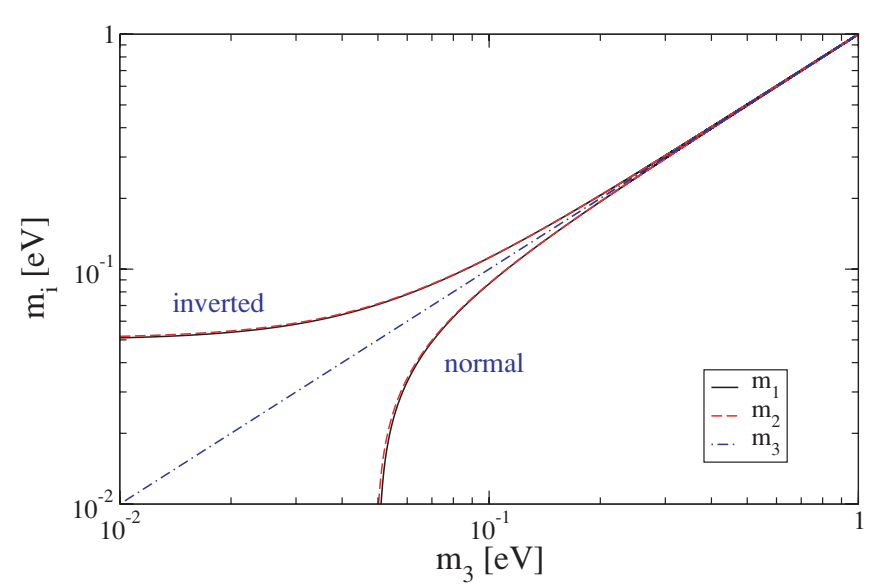

or, for the inverse hierarchy case, as proportional to

$$
n_{\mathrm{IH}}\left(y_{\max }\right)=\int_{0}^{y_{\max }} d y F_{\mathrm{IH}}(y) .
$$

We note that, as stressed in Ref. [20], information on neutrino masses and mixings obtained from neutrino oscillation experiments is actually sufficient in principle to predict $n\left(y_{\max }\right)$ as a function of a single parameter (up to a twofold ambiguity). Thus, in principle, suitably comparing the predicted values of $n\left(y_{\max }\right)$ with results from a future endpoint experiment may end up determining three neutrino masses.

To see how this might work out we make an initial estimate using the best-fit values [6] of neutrino squared mass differences,

$$
\begin{aligned}
& A \equiv m_{2}^{2}-m_{1}^{2}=7.9 \times 10^{-5} \mathrm{eV}^{2}, \\
& B \equiv\left|m_{3}^{2}-m_{2}^{2}\right|=2.6 \times 10^{-3} \mathrm{eV}^{2},
\end{aligned}
$$

and the weighting coefficients,

$$
\begin{aligned}
& \left|K_{11}\right|^{2}=0.67 \\
& \left|K_{12}\right|^{2}=0.29 \\
& \left|K_{13}\right|^{2}=0.04 .
\end{aligned}
$$

Currently $\left|K_{13}\right|^{2}$ is consistent with zero and is only bounded. For definiteness we have taken a value close to the present upper bound. However, we have checked that the effect of putting it to zero is very small. Now, from the two known differences in Eq. (27) we can for each choice of $m_{3}$ (considered as our free parameter) find the masses $m_{1}$ and $m_{2}$, subject to the ambiguity as to whether $m_{3}$ is the largest $(\mathrm{NH})$ or the smallest $(\mathrm{IH})$ of the three neutrino masses. Of course we hope that future long-baseline neutrino oscillation experiments [27-30] might eventually determine whether nature prefers the $\mathrm{NH}$ or the IH scenario.

Figure 3 shows typical solutions for the mass set $\left(m_{1}, m_{2}\right)$ in terms of the free parameter $m_{3}$. Very large values of $m_{3}$ would fall within the sensitivity of upcoming cosmological tests $[10,11]$. In the right panel of Fig. 3 we display the predicted values of $n\left(y_{\max }\right)$ for each possible mass scenario

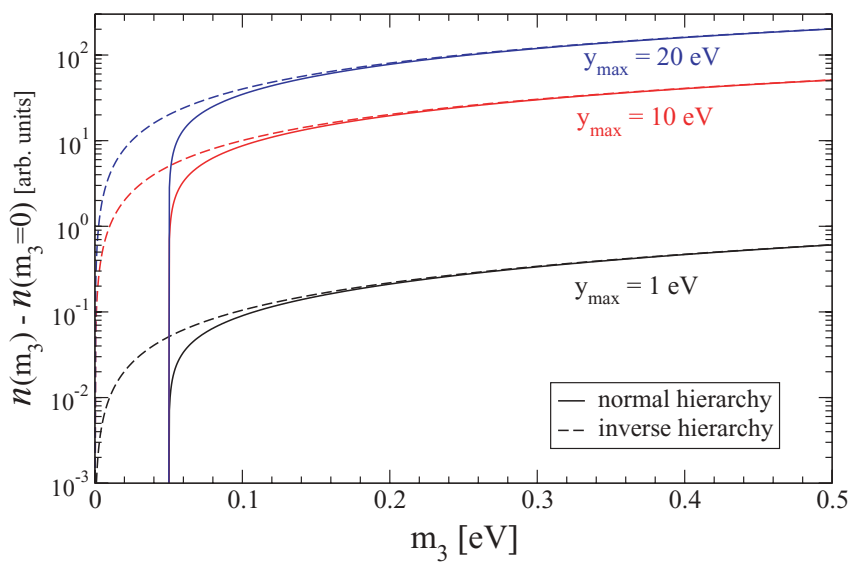

FIG. 3. (Color online) (Left) Typical solutions for $\left(m_{1}, m_{2}\right)$ as a function of $m_{3}$ for the NH case (solid curves) and the IH case (dashed curves); the middle dot-dashed line is given for orientation. (Right) The predictions for the quantities $n\left(y_{\max }\right)$ proportional to the event counting rate, which includes emitted electrons within, respectively, 1, 10, and $20 \mathrm{eV}$ from the appropriate endpoint. 
and the choices of 1,10 , and $20 \mathrm{eV}$ for $y_{\max }$. These quantities are proportional to the electron counting rate in the energy interval from the endpoint (for each mass scenario) to $y_{\max }$ $\mathrm{eV}$ below the endpoint. The different values of $y_{\max }$ reflect, of course, different experimental sensitivities. The main point is that, for sufficiently large $m_{3}$ values, the counting rate is seen to distinguish the different possible neutrino mass sets from each other. We hope that the present method of relating observed neutrino oscillation parameters to predictions for the $\beta$ decay endpoint counting rates may play a useful role in the forthcoming experiments.

\section{SUMMARY AND DISCUSSION}

We have derived the exact relativistic form for the $\beta$ decay endpoint spectrum and presented it in a very simple and useful factorized form. We showed that our exact formula can be well approximated to yield the endpoint form used in the fit method of the KATRIN Collaboration. This was explicitly established through a detailed numerical simulation. We have also discussed the three-neutrino case and shown how information from neutrino oscillation experiments may be useful in analyzing future $\beta$ decay endpoint experiments.

\section{ACKNOWLEDGMENTS}

We are grateful to R. Shrock for helpful discussions. S. S. M. would like to thank the Physics Department at Syracuse University for its hospitality. J. S. would like to express his appreciation for the hospitality received from the AHEP Group of IFIC during the summer of 2005. This work was supported by Spanish grants FPA2005-01269 and the EC RTN network MRTN-CT-2004-503369. The work of J.S. is supported in part by the U.S. DOE under Contract No. DE-FG-02-85ER 40231. S.N. was supported by the DOE Grant DE-FG0297ER41029.
[1] Super-Kamiokande Collaboration, S. Fukuda et al., Phys. Lett. B539, 179 (2002).

[2] SNO Collaboration, Q. R. Ahmad et al., Phys. Rev. Lett. 89, 011301 (2002).

[3] KamLAND Collaboration, T. Araki et al., Phys. Rev. Lett. 94, 081801 (2004).

[4] For a review see T. Kajita, New J. Phys. 6, 194 (2004).

[5] K2K Collaboration, M. H. Ahn et al., Phys. Rev. Lett. 90, 041801 (2003).

[6] For an updated review see the arXiv version hep-ph/0405172 of M. Maltoni, T. Schwetz, M. A. Tortola, and J. W. F. Valle, New J. Phys. 6, 122 (2004) which includes the latest experimental data and references to other groups.

[7] WMAP Collaboration, D. N. Spergel et al., Astrophys. J. Suppl. 170, 377 (2007).

[8] M. Colless et al., Mon. Not. R. Astron. Soc. 328, 1039 (2001).

[9] SDSS Collaboration, M. Tegmark et al., Phys. Rev. D 69, 103501 (2004).

[10] J. Lesgourgues and S. Pastor, Phys. Rep. 429, 307 (2006).

[11] S. Hannestad, Annu. Rev. Nucl. Part. Sci. 56, 137 (2006).

[12] S. R. Elliott and P. Vogel, Annu. Rev. Nucl. Part. Sci. 52, 115 (2002).

[13] KATRIN Collaboration, A. Osipowicz et al., hep-ex/0109033.

[14] V. M. Lobashev, Nucl. Phys. A719, 153 (2003).

[15] C. Kraus et al., Eur. Phys. J. C 40, 447 (2005).
[16] G. Drexlin for the KATRIN Collaboration, Nucl. Phys. Proc. Suppl. 145, 263 (2005).

[17] C. Weinheimer, Nucl. Phys. Proc. Suppl. 168, 5 (2007).

[18] KATRIN Collaboration, J. Angrik et al., FZKA-7090 (KATRIN design report 2004).

[19] A. Monfardini et al., Prog. Part. Nucl. Phys. 57, 68 (2006).

[20] S. S. Masood, S. Nasri, and J. Schechter, Int. J. Mod. Phys. A 21, 517 (2006).

[21] Particle Data Group, S. Eidelman et al., Phys. Lett. B592, 1 (2004).

[22] J. D. Bjorken and S. D. Drell, Relativistic Quantum Mechanics (McGraw-Hill, New York, 1964).

[23] C.-E. Wu and W. W. Repko, Phys. Rev. C 27, 1754 (1983).

[24] A. Saenz, S. Jonsell, and P. Froelich, Phys. Rev. Lett. 84, 242 (2000).

[25] J. Schechter and J. W. F. Valle, Phys. Rev. D 22, 2227 (1980).

[26] Particle Data Group, W. M. Yao et al., J. Phys. G 33, 1 (2006).

[27] C. Albright et al., Report to the Fermilab Directorate, available from http://www.fnal.gov/projects/muon_collider/, hepex/0008064.

[28] M. Apollonio et al., CERN Yellow Report on the Neutrino Factory, hep-ph/0210192.

[29] P. Huber, M. Lindner, and W. Winter, Nucl. Phys. B645, 3 (2002).

[30] Muon Collider/Neutrino Factory, M. M. Alsharoa et al., Phys. Rev. ST Accel. Beams 6, 081001 (2003). 